Original Research Paper

\title{
Technique for In-Vivo Measurements of Heart Deformation Using Digital Image Correlation
}

\author{
${ }^{1}$ Ronald B. Bucinell, ${ }^{2}$ Matthew T. Adams, ${ }^{3}$ Mcolisi Dlamini and ${ }^{4}$ Leo J. Fleishman \\ ${ }^{I}$ Mechanical Engineering, Union College, Schenectady, NY, USA \\ ${ }^{2}$ Research and Advanced Development Group, Bose Corporation, Framingham, MA, USA \\ ${ }^{3}$ Biological and Biomedical Engineerig, McGill University, Montreal, Canada \\ ${ }^{4}$ Biology, Union College, Schenectady, NY, USA
}

Article history

Received: 02-12-2016

Revised: 07-12-2016

Accepted: 14-12-2016

Corresponding Author: Ronald B. Bucinell

Mechanical Engineering,

Union College, Schenectady, NY, USA

Email: bucinelr@union.edu

\begin{abstract}
Many techniques used to measure the deformation of biological tissue are inadequate for measuring deformations of hearts in vivo. Bull Frogs (Rana Catesbeiana) were double-pithed and dissected to expose the amphibian heart for the measurements conducted in this study. White titanium dioxide powder and black charcoal were applied to the surface of the heart to create an artificial surface pattern with high contrast that does not react with the heart muscle or mask surface details. This pattern was then used in conjunction with three dimensional Digital Image Correlation (DIC) to measure full field deformations of the heart through several cardiac cycles. These deformations were measured with a spatial resolution of approximately $0.4 \mu \mathrm{m}$ and temporal resolution of $50 \mathrm{~Hz}$.
\end{abstract}

Keywords: 3D DIC, Heart Deformation, Amphibians, In vivo Measurements, Speckle Pattern

\section{Introduction}

Interest in monitoring deformation of a heart in vivo is partially fueled by cardio vascular disease being the leading cause of deaths in the United States (Mozaffarian et al., 2016). The anisotropic, inhomogeneous and non-linear behavior of the myocardium coupled with its passive and active relaxation and contraction during the cardiac cycle make it difficult to conduct in vitro studies that can replicate the complex system interactions and material responses that occur during in vivo heart deformation.

Lionello et al. (2014; Zhang et al., 2002) report that many standard techniques for evaluating the deformation of biological specimens are difficult to adapt to in vivo measurements because they are limited to small strains, require contact with the specimen, or do not have appropriate spatial resolution. One technique that is not limited to small strains, is non-contact and can provide appropriate spatial resolution in all three dimensions is the Stereo (or 3D) Digital Image Correlation technique (DIC) (Sutton et al., 2009).

Critical to the successful application of the DIC technique is having either a natural occurring or artificial stochastic pattern on the surface of the specimen. This pattern should consist of high contrast components (background and speckles), should not react with the specimen, should adhere to the specimen and should not mask the details of the underlining deformations. The speckles size in the stochastic pattern should be larger than three pixels in diameter to avoid aliasing issues during correlation and care should be taken to minimize the speckle size to avoid compromising spatial resolution. In vivo heart studies complicate pattern selection because the heart is wet, subject to large-rapid deformations and the cardiac cycle is controlled by an electro-chemical process in the myocardium that could be impacted by the materials used to pattern the surface.

Zhang et al. (2002; Miri et al., 2012; Lionello et al., 2014; Affagard et al., 2015) have all successfully used $2 \mathrm{D}$ DIC on both hard and soft in vitro biological specimens. 2D DIC is limited to a single plane that has to be parallel to the sensor or parallax can occur in the recorded image. Bucinell et al. (2010) successful used 3D DIC to characterize complex three dimensional load paths in primate mandibles subject to in vitro loads.

Gaudette et al. (2001) and Chiang (2010) used 2D DIC to characterize in vitro myocardial tissue of rabbit hearts. In their study they were able to show that the 2D 
DIC results were comparable to sonomicrometry results. Hokka et al. $(2015$; 2016) were the only reported 3D DIC in vivo deformation measurements of a human heart during surgery. It should be noted that the natural pattern of the heart was used for DIC correlation causing severe limits in spatial resolution. Even with poor spatial resolution, the DIC results were used to assist surgeons in assessing the success of the surgery prior to closing the thoracic cavity.

Further development of the spatial and temporal resolution of the 3D DIC techniques for in vivo heart deformation studies have to be conducted on species other than humans because of ethical and regulatory issues, this study uses Bull Frogs (Rana Catesbeiana). The primary difference between a frog heart and a human heart is that the frog heart has two atria and one ventricle, while the human heart has two atria and two ventricles. However, the frog and human hearts do have similar myogenic mechanisms for controlling the cardiac cycle. One advantage of using a frog for in vivo heart studies is that the difficulties associated with anesthetizing mammals are avoided when frogs are pithed.

The remainder of this paper will describe a 3D DIC technique that was successfully used to measure in vivo heart deformations in a frog with high spatial and temporal resolution.

\section{Materials and Methods}

The following subsections will cover the specimen preparation, stochastic pattern generation, lighting and the 3D DIC configuration.

\section{Specimen Preparation}

In this study Bull Frogs (Rana Catesbeiana) were used in the experiments. In preparation for heart observation the frogs was first cooled to ensure it was serene and remained in a normotensive state prior to the experiments. The frogs were then double-pithed following protocols established and approved by the Union College Institutional Animal Care and Use Committee (IACUC). This procedure destroys the brain and spinal cord, immobilizes the frog and renders the nervous system insensitive. In this state the circulatory system continues to operate rhythmically for up to one hour in the absence of nervous system input.

Post pithing the frogs were placed on a dissection tray exposing their ventral side. The dissection started with a midline cut from the bottom of the abdomen to the bottom of the jaw. "T" cuts were made at the base of the jaw and the bottom of the abdomen to fully expose the thoracic cavity. The skin flaps were pinned back using dissecting pins so that measurements could be taken without obstruction.

\section{Stochastic Pattern Generation}

Critical to the DIC technique is the application of a stochastic pattern on the surface of the specimen. In these experiment the pattern is being placed on a heart in vivo, therefore it is critical that the materials used dose not react with the electro-chemical process in the myocardium that control the cardiac cycle. Unsuccessful attempts were made to generate the stochastic pattern using both acrylic and latex paints. The paints either had difficulty adhering to the heart, were too thick to enable an unobstructed views of the deformation, or in some instances reacted with the heart causing it to stop beating.

The technique used by Gaudette et al. (2001) and Chiang (2010) to produce the stochastic pattern was to disperse small silicon carbide particles (approx.. $40 \mu \mathrm{m}$ ) on the surface of the heart which created a dark speckle pattern on the light tissue surface. This technique worked primarily because the experiments were performed in vitro and the pumping action was being generated by a latex balloon inside the heart. In this study the in vivo heart surface changes color dramatically as blood is pumped through it. Our attempt to use this technique lead to an unsuccessful result.

It became clear that the heart needed to be coated with one color with a contrasting color being used as speckles. Because the sensors being used only recorded gray scale images, the two contrasting colors chosen were white and black. First chalk dust and charcoal shavings were tested to see if they were appropriate for applying the stochastic pattern. The moist environment around the heart caused the chalk dust to dissolve again leading an unsuccessful result.

Finally, titanium dioxide powder was applied to the surface of the heart as the base and charcoal powder was used to form the speckles. These contrasting powders proved successfully in place a durable stochastic pattern on the surface of the heart that gave an appropriate speckle size, did not react with the heart and did not mask the details of the hearts deformation during the cardiac cycle. Additionally, the pattern could be easily removed from the heart by gently rubbing a moist gauze pad over the specimen. This was necessary replace the pattern on the heart.

As will be discussed in one of the following section, the sensor being used for these experiments were 1280 px by 1024 px photo arrays. The frog hearts used in these experiments fit into a $15 \mathrm{~mm}$ diameter circle, so a $25 \times 20 \mathrm{~mm}$ field of view was calibrated. This corresponds to $19.5 \mu \mathrm{m}$ per pixel. The stochastic pattern speckle size needs to be greater than 3 pixels in diameter to avoid aliasing, but must be minimized to maximize spatial resolution. These requirements lead an optimal charcoal particle size of around $60 \mu \mathrm{m}$. To achieve this 
particle size, powdered charcoal was crushed to the appropriate size in a pestle and mortar. The crushed charcoal powder was then placed in a desiccator for a minimum of $24 \mathrm{~h}$ prior to application to insure the particles did not clump together during the speckle application procedure.

The as-purchased titanium dioxide powder was also crushed in a pastel and mortar to reduce the size of the powder so it would not mask the details of the hearts deformation once applied. The crushed powder was also placed in a desiccator for a minimum of $24 \mathrm{~h}$ prior to application to make the powder easier to apply to the heart in a uniform manner.

The application of the stochastic pattern to the heart started by placing a small amount of the dried titanium dioxide powder on a sheet of paper. The paper was held just above the dissected frog while a pipette was used to slowly blow a thin coating of powder over the surface of the heart. Care was taken to insure the final surface coating of powder was uniform and thin.

The next step in the process of generating the stochastic pattern on the surface of the heart is applying the charcoal particles that will make up the speckles. This process starts by placing a small amount of charcoal powder on a piece of paper. The paper is held over the dissected frog and a pipette is used to create a small cloud of charcoal powder over the thoracic cavity. After the cloud falls and settles on the surface of the heart, the process is repeated until an appropriate density of charcoal particles in on the surface.

Figure 1 shows a stochastic pattern that has been placed on the surface of a heart using the procedure outlined above. The application of the stochastic pattern in vivo requires considerable practice to master. Training in this technique was performed using damp tofu to avoid the unnecessary dissection of frogs.

\section{Lighting}

The complete cardiac cycle for a frog is about 1 to 2 sec long. To capture the complex motion associated with the deformation of the heart during a cardiac cycle a frame rate of 50 frames per second was used in this study to avoid blurring of the images recorded by the photo sensors. This frame rate requires the surface to be illuminated with intense light which also gave rise to glare on the surface.

Several lighting sources were considered for this study. Direct incandescent, fluorescent, halogen and light-emitting diodes at intensity levels that would properly illuminate the heart were all found to generate enough heat on the surface of the heart to alter the normal cardiac cycle. Fiber optic illuminators with a quartz halogen light source (cool lighting system, Dolan-Jenner Fiber Lite DC-950) were found to provide light at the property intensity without heating of the surface.
The fiber optic illuminators still generate glare on the surface of the heart. For this application glare was minimized by placing linear polarizing filters on the light sources and lenses of the photo sensors. The intensity of the fiber optic illuminators were increased to compensate for the reduction in light intensity on the surface of the heart that resulted from the use of the filter.

\section{D DIC Configuration}

The experiments set-up used in this study is shown in Fig. 2. Two Basler A504 (Basler Vision Technologies) high speed cameras were mounted above the specimen on a tripod with an extension equipped with two axis positioning stages. Each of these cameras were equipped with a $50 \mathrm{~mm}$ Unifoc Schneider Componon-S 2.8/50 lens. The cameras were controlled by the ARAMIS DIC System (GOM $\mathrm{mbH}$, Braunschweig, Germany).

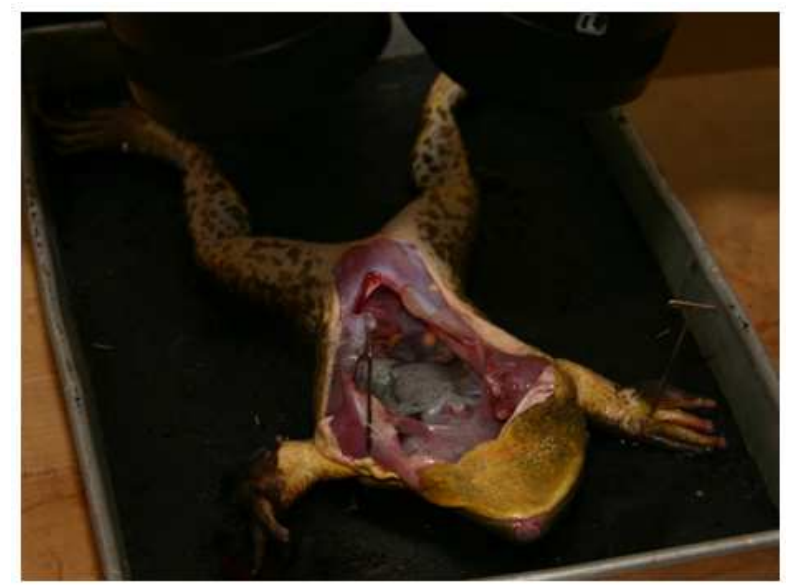

Fig. 1. The stochastic pattern created using titanium dioxide powder and powder charcoal on the surface of the dissected frog heart

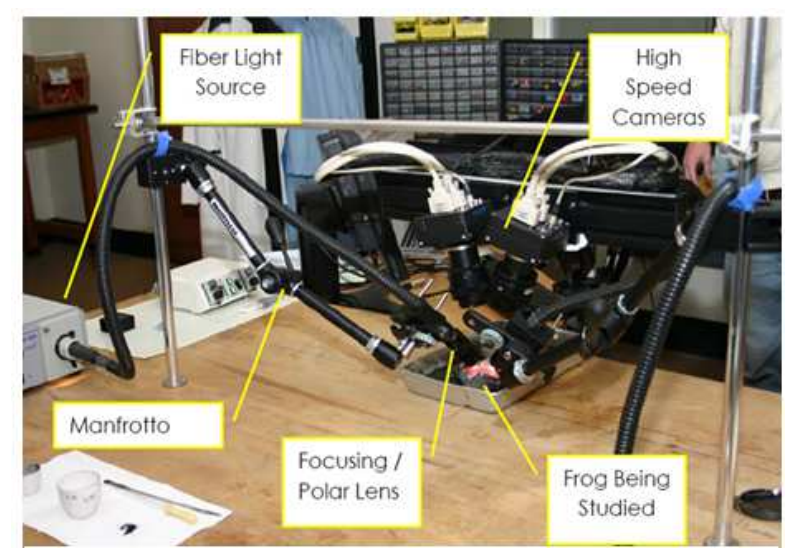

Fig. 2. The experimental set-up used in this study including the high speed DIC photo sensors, lighting and specimen 
The DIC sensors in the Basler cameras were $1280 \mathrm{px}$ by $1024 \mathrm{px}$. The field of view of the cameras was calibrated to $25 \mathrm{~mm}$ (x) by $20 \mathrm{~mm}$ (y) $10 \mathrm{~mm}$ (zdepth of field). This results in a pixel dimension of $19.5 \mu \mathrm{m}$. The system was calibrated to 0.02 pixels of displacement or approximately $0.4 \mu \mathrm{m}$ displacement. The cameras were then set to take 50 frames per second with a shutter speed of $10 \mathrm{~ms}$.

The two fiber optic light sources were held in position over the specimen using Manfrotto Magic Arms.

\section{Results}

Figure 3 shows the out-of-plane displacements (Displacement $\mathrm{Z}$ ) on the surface of the heart, overlaid on the heart, at four point in the cardiac cycle (A, B, $\mathrm{C}, \mathrm{D})$. The strip chart below the images tracks point displacements through three cardiac cycles at the location of the red $(\bullet)$, blue $(\bullet)$ and black $(\bullet)$ dots in

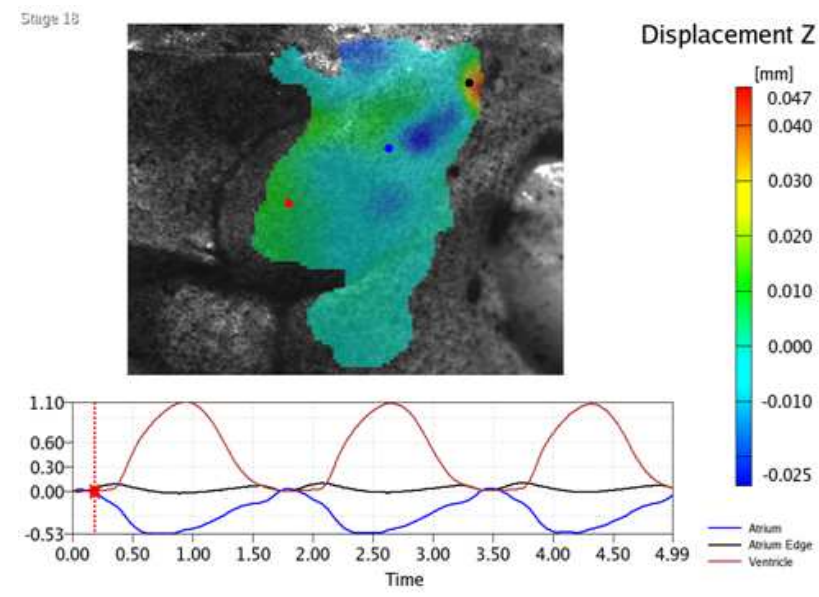

(A)

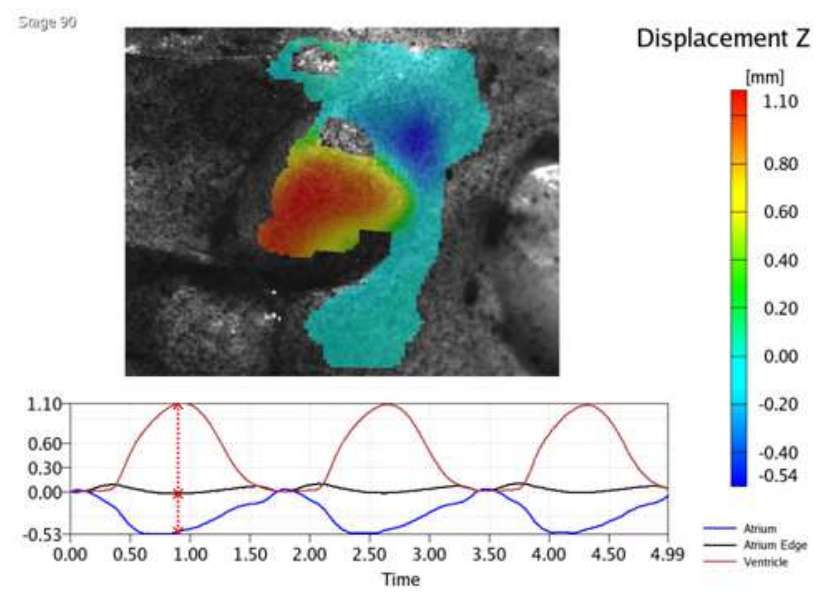

(C)
Fig. 3A. These three dots are respectively placed on the ventricle, lower atrium and upper atrium. All displacements are in millimeters. The red vertical line on the strip chart shows the location in the cardiac cycle where the image above was taken. The scale to the right of the image is for the overlaid $\mathrm{z}$ displacement field shown in the image. Displacement fields in the $\mathrm{x}$ and $\mathrm{y}$ (in the plane of the image) could also have been produced for the cardiac cycle, but were not included in this study.

The 3D DIC technique uses the stochastic pattern to calculate the deformations on the surface of the heart in the $\mathrm{x}, \mathrm{y}$ and $\mathrm{z}$ directions. These deformations are used in continuum mechanics to compute surface strains. Figure 4 shows the Major (Maximum Principle) strain calculated using the displacement field of the heart at the same time as the $\mathrm{z}$ displacement in Fig. 3B. The scale to the right of the image is in percent strain.

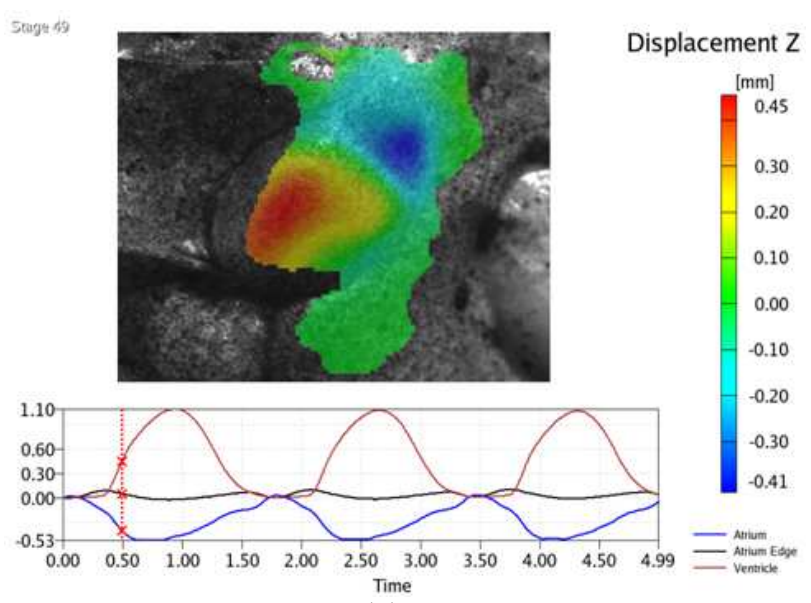

(B)

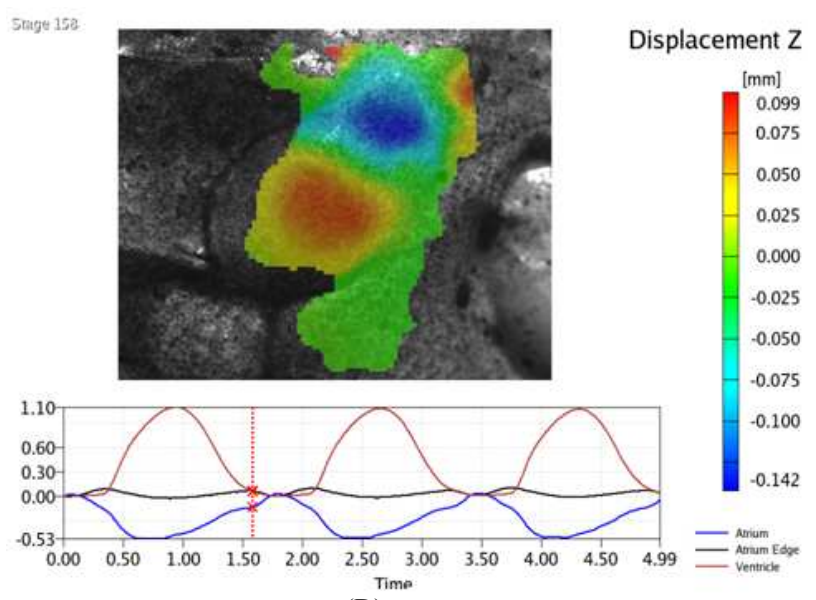

(D)

Fig. 3. A sequence of images of the out-of-plane displacement $(z)$ overlaid on the patterned heart at various points in the cardiac cycle 


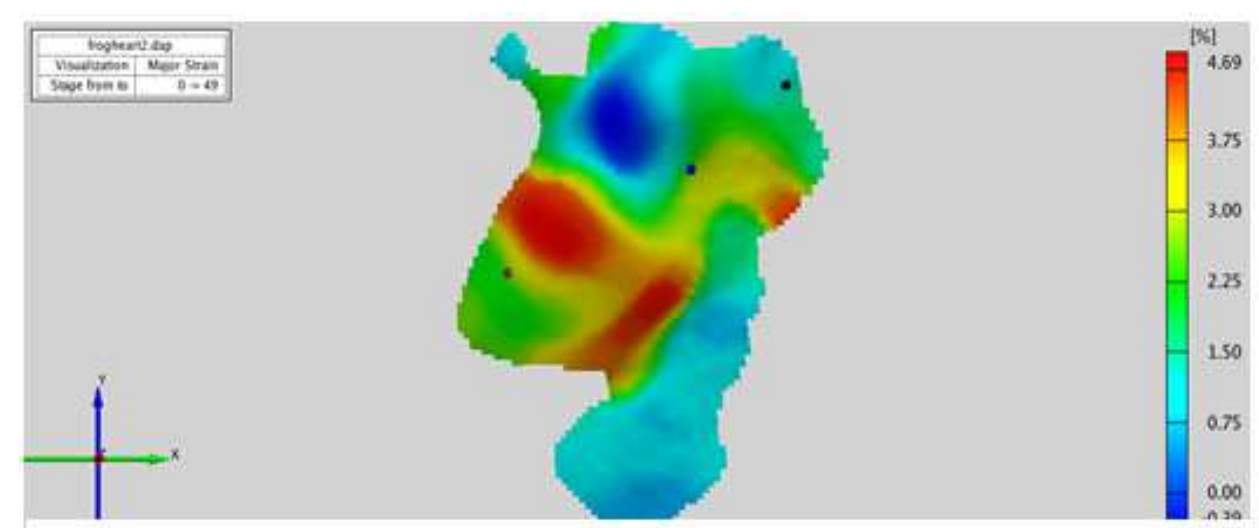

Fig. 4. Map of the major (maximum principal) strains on the surface of the heart at the same point in the cardiac cycle as the $\mathrm{z}$ displacements in Fig. 3B

\section{Discussion}

The advantage of $3 \mathrm{D}$ DIC over $2 \mathrm{D}$ DIC is that deformation measurements out of the plane of the sensors on the surface of the specimen can be measured. Because this is a white-light technique, the out-of-plane deformation measurement is limited. This limitation is a result of the lenses having a singular point of focus. As objects move away from this point they go out of focus. The algorithm used to track the deformation of the speckle pattern can tolerate only a limited amount of optical aberration. This limit controls the depth of field, which is calculated and reported during calibration of the 3D DIC system.

The 3D DIC technique captures the complex deformation on the surface of the heart as it progresses through the cardiac cycle. The results of this study showed that different regions of the heart expand and contract at different times during the cycle. Being able to capture this detail requires a proper stochastic pattern to insure spatial resolution and the appropriate shutter time settings to insure temporal resolution.

The generation of the stochastic pattern is more of an art than a science. The procedure outlined above requires a great deal of practice to master. This is why training is conducted on moist tofu. Using this analog allows the researcher to become familiar with the application technique and gives them a sense of what patterns will work and which will not. After the tofu was speckled it was be placed under the $3 \mathrm{D}$ DIC system shown in Fig. 2 and two images of the surface were taken. These images were then correlated by the system. If the pattern was good, displacement fields were calculated which indicate the error of the measurements. If the pattern was not good, no displacement field was calculated or a partial displacement field was calculated. Poor patterns typically resulted from lack of speckle density or not enough contrast in between the speckles and the background. Use of tofu enabled the technique to be mastered and reinforced using a tactile approach.

Imaging techniques require objects to have limited deformation while the camera shutter is open and the sensor is recording. This typically means that the object cannot move more than a pixel during this time. The shutter time is also a function of light intensity. In this study light intensity was limited by fiber optic light sources having polarizing filters placed on them to minimize glare. Seeking out the proper shutter time was a result several trials that started at the lower shutter time limit and progressed to longer times until the minimum shutter time that would produce an image was found.

The time between images was also determined through trial and error. The cameras used in this study were capable of up to 500 frames per second at full field and 8000 frames per second at partial field. The number of frames taken during the cardiac cycle means longer computation times. The minimum number of frames per second that would provide the adequate detail deformations details was determined through several trials. 50 frames per second appeared to be the appropriate balance between temporal resolution and computational intensity.

\section{Conclusion}

The 3D DIC technique has been shown to be a robust technique for measuring the complex deformations on the surface of a heart in vivo. Turnkey systems are widely available to the researcher to control cameras, calibrate the field of view and correlate the results. Special care needs to be taken in applying a stochastic pattern to the surface to avoid aliasing, maximize spatial resolution and make sure the materials being used do not react with the electro-chemical process in the myocardium. Special care needs to be taken when choosing lighting to make sure the intensity is high 
enough to accommodate shutter times and to minimize glare on the specimen's surface.

The detailed three dimensional deformations and strains measured on the surface of the heart using 3D DIC can be used as both a research tool and diagnostic tool.

\section{Acknowledgement}

Union College internal research funds were used to purchase specimens and supplies for the experiments discussed in this study. The DIC system used in the experiments described in this study was purchased through a grant from the National Science Foundation (NSF-MRI 0116494).

\section{Funding Information}

This manuscript was not prepared using any external funding sources.

\section{Author's Contributions}

Ronald B. Bucinell: Trained Adams and Dlamini in the use of the DIC technique, developed the protocols used to collect the DIC data, supervised Adams and Dlamini and wrote the manuscript.

Matthew T. Adams: Performed dissection on specimens, performed experiments and performed data reduction.

Mcolisi Dlamini: Performed dissection on specimens, performed experiments and performed data reduction.

Leo J. Fleishman: Trained Adams and Dlamini on dissection techniques, pithed all specimens, assisted in preparing specimens for experiments and provided amphibian anatomical expertise.

\section{Ethics}

All amphibians used in the experimental described in this paper were cared for using protocols set up and approved by the Institutional Animal Care and Use Committee (IACUC) at Union College which complies with the animal research guidelines of the United States National Institutes of Health.

No part of this manuscript is being considered for publication in whole or in part elsewhere. The corresponding author affirms that all of the authors have read and approved the manuscript.

\section{References}

Affagard, S.J., P. Feissel and S.F. Bensamoun, 2015. Measurement of the quadriceps muscle displacement and strain fields with ultrasound and Digital Image Correlation (DIC) techniques. IRBM, 36: 170-177. DOI: 10.1016/j.irbm.2015.02.002
Bucinell, R.B., D.J. Deagling, W.S. McGraw and A. Rapoff, 2010. Full-field characterization of wishboning strain in the colobine mandibular symphysis. Anatomical Record, 293: 549-556. DOI: $10.1002 /$ ar.21120

Chiang, F.P., 2010. Soft tissue characterization at macro to nano scales using digital speckle photography. Proceedings of 11th Pan-American Congress of Applied Mechanics, Jan. 4-8, Foz do Iquacu, PR, Brazil.

Gaudette, G.R., J. Todaro, I.B. Krukenkamp and F.P. Chiang, 2001. Computer aided speckle interferometry: A technique for measuring deformation of the surface of the heart. Ann. Biomed. Eng., 29: 775-780. DOI: $10.1114 / 1.1397785$

Hokka, M., N. Mirow, H. Nagel, M. Irqsusi and S. Vogt et al., 2015. In-vivo deformation measurements of the human heart by $3 \mathrm{D}$ digital image correlation. J. Biomechan., 48: 2217-2220. DOI: 10.1016/j.jbiomech.2015.03.015

Hokka, M., N. Mirow, H. Nagel, S. Vogt and V.T. Kuokkala, 2016. DIC Measurements of the Human Heart during Cardiopulmonary Bypass Surgery. Mechan. Biol. Syst. Mater., 6: 51-59. DOI: 10.1007/978-3-319-21455-9_6

Lionello, G., C. Sirieix and M. Baleani, 2014. An effective procedure to create a speckle pattern on biological soft tissue for digital image correlation measurements. J. Mecah. Behav. Biomed. Mater., 39: 1-8. DOI: 10.1016/j.jmbbm.2014.07.007

Miri, A.K., F. Barthelat and L. Mongeau, 2012. Effects of dehydration on the viscoelastic properties of vocal folds in large deformations. J. Voice, 26: 688-697. DOI: 10.1016/j.jvoice.2011.09.003

Mozaffarian, D., E.J. Benjamin, A.S. Go, D.K. Arnett and M.J. Blaha et al., 2016. Heart disease and stroke statistics-2016 update. American Heart Association, Dallas, Tx, USA.

Sutton, M.A., J.J. Orteu and H.W. Schreier, 2009. Image Correlation for Shape, Motion and Deformation Measurements. 1st Edn., Springer, New York, ISBN: 038778747X, pp: 322.

Zhang, D., C.D. Eggleton and D.D. Arola, 2002. Evaluating the mechanical behavior of arterial tissue using digital image correlation. Exp. Mechan., 42: 409-416. DOI: $10.1007 / \mathrm{BF} 02412146$ 